\title{
AS CHUVAS NA CIDADE DO RECIFE: UMA CLIMATOLOGIA DE EXTREMOS
}

\author{
WANDERLEY, Lucas Suassuna de Albuquerque - lucassaw.13@gmail.com \\ Universidade Federal de Pernambuco / UFPE
}

NÓBREGA, Ranyere Silva - ranyere.nobrega@yahoo.com.br Universidade Federal de Pernambuco / UFPE

MOREIRA, Ayobami Badiru - ayo.badiru@hotmail.com

Universidade Federal de Pernambuco / UFPE

ANJOS, Rafael Silva dos - anjos.rsa@gmail.com

Universidade Federal de Pernambuco / UFPE

\section{ALMEIDA, Caio Américo Pereira de - caioamerico@gmail.com Universidade Federal de Pernambuco / UFPE}

\begin{abstract}
RESUMO: O trabalho tem como objetivo investigar as frequências, as tendências e o tempo de recorrência dos eventos pluviométricos extremos na cidade do Recife, Pernambuco, Brasil. Para a análise foram utilizados dados de precipitação diária no período de 1961 a 2016. Aplicou-se a técnica dos Quantis para determinar os eventos diários extremos, classificados nas categorias de chuvas: extremamente fracas, muito fracas, fracas, habituais, fortes, muito fortes e extremamente fortes. Para avaliar a tendência das frequências de ocorrência anual dos eventos extremos diários de precipitação, foi aplicado o teste de Mann-Kendall; e, para estimar o tempo de retorno, foi utilizada a distribuição de Gumbel. A investigação das frequências anuais dos eventos de precipitações extremas diárias, pelo método de Mann-Kendall, não demonstrou tendências significativas. A distribuição de Gumbel estimou satisfatoriamente a probabilidade de ocorrência e o tempo de retorno de eventos pluviais extremos, além disso, esse modelo de distribuição reforçou a suscetibilidade da área de estudo aos eventos intensos de chuvas diárias. Portanto, a recorrência dos eventos pluviais extremos deve ser considerada um importante aspecto para o planejamento territorial da cidade de Recife.
\end{abstract}

PALAVRAS-CHAVES: Eventos extremos, Chuva, Tempo de retorno.

THE RAINFALL AT THE CITY OF RECIFE: A CLIMATOLOGY OF EXTREMES

ABSTRACT: The aim of this paper is to investigate the extreme rainfall events frequencies, trends and recurrence interval in the city of Recife, Pernambuco, Brazil. For the analysis proposed, daily precipitation data were used for the period from 1961 to 2016.The Quantiles technique was used to determine the extreme daily events which were classified in the following categories: extremely weak, very weak, weak, habitual, strong, very strong and extremely strong rainfall. To evaluate the tendency of annual occurrence frequencies of daily extreme events of precipitation it was applied the MannKendall test. And to estimate the time of return the Gumbel distribution was used. The investigation of the annual frequencies of extreme daily precipitation events by the Mann-Kendall method did not show significant trends. The Gumbel distribution estimated satisfactorily the probability of occurrence and the time of return of extreme rainfall events, in addition, this distribution model reinforced the susceptibility of the area to the intense events of daily rains. Therefore, the recurrence of extreme rainfall events should be considered as an important aspect for the territorial planning in the city of Recife.

KEYWORDS: Eextreme events, Rainfall, time of return. 


\section{INTRODUÇÃO}

A variabilidade espaço-temporal da precipitação é uma das principais características dos climas tropicais. Nesses domínios climáticos, os eventos extremos de chuva, associados às formas de ocupação do espaço pelo homem, dão origem a diversos problemas de ordem socioambiental. Em parte, essa desarmonia entre o clima e as feições culturais do espaço pode ser atribuída a não aplicação, no planejamento territorial, da noção de ciclicidade dos fortes episódios de chuva. Em muitos casos, enfatizam-se os conceitos de "anomalia ou fatalidade climática" em detrimento dos ciclos de ocorrência dos eventos pluviométricos.

Estudos recentes têm se preocupado em investigar a frequência, a tendência e o tempo de retorno dos eventos extremos de precipitação, apresentando métodos eficientes na caracterização de episódios pluviométricos. Dentre esses estudos, destacam-se Silva et al. (2013), que utilizaram a distribuição de Gumbel para estimar o tempo de retorno de eventos máximos de precipitação na cidade do Recife. Zahiri et al. (2016) utilizaram essa mesma distribuição para analisar o tempo de retorno de eventos diários de precipitação na África Ocidental, e Zhang et al. (2011) avaliaram a tendência de eventos extremos de chuva na região da bacia do rio Yangtze (China). Khoi et al. (2016) identificaram, através do teste não paramétrico de Mann-Kendall, tendências de aumento nos volumes de precipitação no noroeste da cidade de Ho Chi Minh (Vietnã), e diminuição dos volumes no sudeste da referida área urbana. Farias et al. (2012) e Duarte et al.(2015) classificaram eventos extremos de precipitação no estado de Pernambuco, Brasil, a partir da técnica dos Quantis no intuito de relacioná-los aos desastres decorrentes.

Nos ambientes urbanos, as inundações causadas por chuvas intensas são os impactos mais comuns ocasionados por eventos climáticos. Por esta razão, conhecer o comportamento dos extremos de chuva é essencial para a gestão da drenagem e planejar a infraestrutura das cidades (ARNBJERG-NIELSEN et al., 2013; GOCIC et al., 2013; KHOI et al., 2016). Nas cidades brasileiras, de maneira especial, o tratamento fatalista empregado aos eventos extremos de precipitação resulta em graves problemas estruturais, pois as cidades, de uma maneira geral, não são planejadas para conviver com volumes pluviométricos de alta intensidade.

Nesse contexto, a cidade do Recife, localizada no litoral oriental do Nordeste Brasileiro (NEB), enfrenta problemas históricos relacionados a eventos pluviais intensos sobre áreas de infraestrutura inadequada. As chuvas intensas são um aspecto importante do clima da Região Metropolitana do Recife, pois apresentam grande percentual dos totais mensais anuais de precipitação. Souza et al. (2012) verificaram que volumes diários de chuva superiores a $55 \mathrm{~mm}$, registrados no sítio urbano do Recife, estão frequentemente relacionados a deslizamentos de encosta, alagamentos e inundações. Além disso, esses autores observaram que, apesar das precipitações diárias intensas ocorrerem com maior frequência entre os meses de março a agosto, são registrados grandes volumes fora do período de maior concentração das chuvas.

A respeito da gênese dos sistemas atmosféricos produtores de eventos pluviométricos intensos, Machado et al. (2012) observaram que Distúrbios Ondulatórios de Leste (DOLs) quando associados a desvios positivos de temperatura das águas do mar, próximas a costa do NEB, podem modular a 
intensificação de sistemas convectivos responsáveis pela produção de precipitações intensas. Os DOLs podem ser classificados como sistemas atmosféricos de escala sinótica que ocasionam perturbações nos campos de vento e de pressão com deslocamento de leste para oeste, no sentido dos ventos alísios (MOTA, 1997).

Essas perturbações ondulatórias podem ser desencadeadas por mecanismos diferenciados ao longo do ano. Entre os meses de julho a agosto a convergência de ventos provindos de sistemas frontais com os alísios, é apontada como principal gatilho de formação dos DOLs. De outro modo, uma maior atividade da Zona de Convergência Intertropical (ZCIT) também é apontada como um mecanismo de formação desses distúrbios. Além disso, a confluência dos DOLs com a brisa terrestre, que ocorre nas proximidades da costa leste do NEB à noite e nas primeiras horas da manhã, intensifica a convecção e pode ocasionar totais pluviométricos superiores a $100 \mathrm{~mm}$ por dia (MOLION e BERNARDO, 2002).

A interação dos eventos pluviais extremos com outros condicionantes do sistema urbano da cidade do Recife pode gerar consequências múltiplas. A depender das combinações climáticas cíclicas, as chuvas registradas sobre a cidade podem variar em mais de $50 \%$ acima da média histórica, acarretando, conforme a parte da paisagem urbana afetada, em respostas distintas: alagamentos e enchentes-relâmpago na planície e "movimentos de massa" nos morros (CORREA, 2010).

Apesar da importância do tema, Barros et al. (2013) destacam que há poucos estudos sobre o clima da cidade do Recife, e que aqueles existentes enfatizam a temática da variabilidade. Nesse viés, a incorporação do tratamento cíclico, nos estudos climáticos, pode funcionar como uma importante ferramenta do planejamento territorial de prevenção aos desastres associados aos eventos extremos (ZAHIRI et al.,2016).

Diante disso, no intuito de contribuir com os conhecimentos a respeito da climatologia das chuvas na cidade do Recife, o presente estudo objetiva investigar as frequências, as tendências e o tempo de recorrência dos eventos pluviométricos extremos.

\section{MATERIAIS E MÉTODOS}

\section{LOCALIZAÇÃo DA ÁREA DE ESTUDO}

A cidade do Recife está localizada no estado de Pernambuco, no setor oriental da Região Nordeste do Brasil (Figura 01). É a maior área urbana do estado, banhada a leste pelo Oceano Atlântico e limitada pelos municípios de Olinda, Paulista, Camaragibe, Cabo de Santo Agostinho, Jaboatão dos Guararapes e São Lourenço da Mata. 

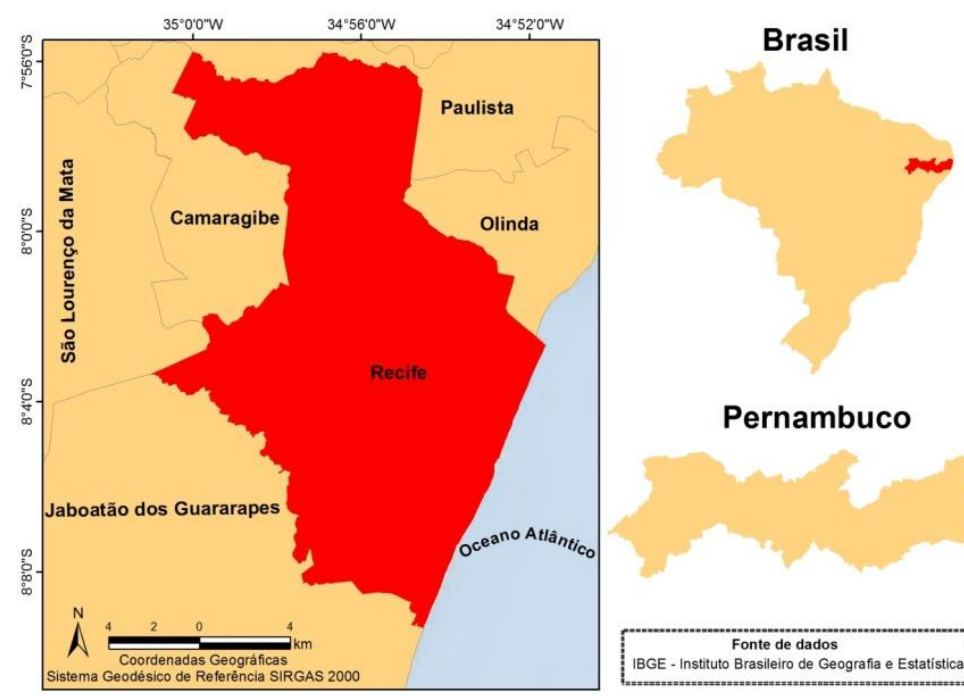

Pernambuco

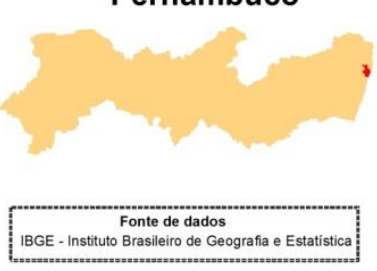

Figura 1 - Localização do município do Recife e da estação do INMET. Org. dos autores.

\section{MATERIAIS}

Os dados diários de precipitação (1961-2016) utilizados no estudo foram adquiridos na base de dados do Instituto Nacional de Meteorologia (INMET). Os dados são referentes à estação pluviométrica do Curado (Recife/PE), a qual possui coordenadas de -34,95 (Longitude) e -8,05 (latitude), e altitude de $10 \mathrm{~m}$ (Figura 01).

\section{MÉTODOS}

A primeira etapa de pesquisa consistiu na aplicação da técnica dos Quantis (Xavier, 2002), sobre a série pluviométrica, para classificar os eventos extremos diários de precipitação. Consideradas probabilidades contínuas relacionadas à determinada variável aleatória $X$ (chuva), pode-se definir o quantil (Qp) para cada número real entre zero e um, respondendo à seguinte condição:

$$
\operatorname{Prob}(X \leq Q p)=p
$$

Nessa perspectiva, os eventos pluviométricos diários foram agrupados em oito classes (Tabela 01), considerando os seguintes valores: $p=0,05 ; p=0,15$; $p=0,35 ; \quad p=0,65 ; \quad p=0,85 ; \quad p=0,95$, e classificando-as como chuva extremamente fraca, muito fraca, fraca, habitual, forte, muito forte e extremamente forte. Essa proposta adaptou as subdivisões quantílicas propostas por Duarte et al. (2015). 
Tabela 1 - Classes e intervalos dos quantis de precipitação diária na cidade do Recife entre os anos de 1961-2016.

\begin{tabular}{ll}
\hline Classes Quantilicas & Intervalos Quantílicos \\
\hline Extremamente fraca & $\mathrm{Qp} \leq 0,05$ \\
Muito fraca & $0,05<\mathrm{Q} p \leq 0,15$ \\
Fraca & $0,15<\mathrm{Q} \leq 0,35$ \\
Habitual & $0,35<\mathrm{Q} \leq 0,65$ \\
Forte & $0,65<\mathrm{Q}-0,85$ \\
Muito forte & $0,85<\mathrm{Q} p \leq 0,95$ \\
Extremamente forte & $\mathrm{Qp}>0,95$ \\
\hline
\end{tabular}

Org. dos autores.

A segunda etapa teve como objetivo analisar as frequências de ocorrência mensal e anual das classes quantílicas muito forte e extremamente forte (representadas pelos valores de 25,1 $\mathrm{mm}$ e 50,8 $\mathrm{mm}$, respectivamente), aplicadas aos eventos diários de chuva. Optou-se por analisar também a frequência de eventos excepcionais, iguais ou superiores a $100 \mathrm{~mm}$ $(2 * \mathrm{Qp}=0,95)$. Como método complementar, utilizou-se o teste de Mann-Kendall para investigar possíveis tendências das frequências de ocorrência (mensal e anual) dos eventos extremos e das distribuições dos valores de chuvas mensais e anuais.

Por fim, foi aplicado o teste de Gumbel para estimar o tempo de recorrência e a probabilidade anual de ocorrência dos eventos máximos de chuvas. A distribuição foi aplicada sobre os dados máximos diários registrados por ano, e sobre os dados máximos diários registrados por mês para os anos de 1961 a 2016. A respeito deste método, Sansigolo (2008), ao comparar as mais frequentes distribuições utilizadas, considera que a de Gumbel é a mais adequada para descrever e extrapolar extremos de precipitação diária para longos períodos de retorno. A função de densidade de probabilidade acumulada pode ser descrita como:

$$
F(x)=\exp (-\exp (-(x-a) / b))
$$

Onde $a$ e b são parâmetros do modelo de distribuição, e $x$ é denominado de máxima variável aleatória de precipitação. A distribuição é expressa então da seguinte meneira:

$$
F(x)=\exp (-\exp (-u)) \text { e } u=-\ln (-\ln (F(x)))
$$

Onde u é definido como uma variável reduzida de Gumbel, sendo $u=(x-$ a)/b.

A aderência da distribuição de Gumbel à série pluviométrica estudada foi testada pelo método de Kolgomorov-Smirnov. Através desse método, comparase a máxima divergência entre a probabilidade de ocorrência dos eventos extremos calculada pela distribuição de Gumbel e a probabilidade empírica. 


\section{RESULTADOS E DISCUSSÃO}

\section{CLIMATOLOGIA DA PRECIPITAÇÃO}

A cidade do Recife está inserida em um contexto climático tropical úmido, com chuvas concentradas durante o outono e o inverno. Nesta perspectiva, de acordo com Mendonça e Dani-Oliveira (2007), o clima da área de estudo é classificado como Clima Tropical Litorâneo do Nordeste Oriental, o qual se diferencia dos demais climas mais secos do interior da região Nordeste.

A média pluviométrica anual registada na estação do INMET é de 2292,9 $\mathrm{mm}$, com um desvio padrão de $482,5 \mathrm{~mm}$. A distribuição média mensal da chuva (Figura 02(a)) é caracterizada por uma maior concentração entre os meses de abril a agosto, sendo os meses de maio a julho aqueles com maior volume médio.

A Figura 02(b) referente aos valores anuais de precipitação entre os anos de 1961 e 2016 revela uma considerável variabilidade anual da precipitação, com alguns anos em que as chuvas superaram os 3000 mm (1964, 1986, 2000 e 2011) e outros em que os volumes foram inferiores a $1500 \mathrm{~mm}(1993,1998 \mathrm{e}$ 1999).

(a)
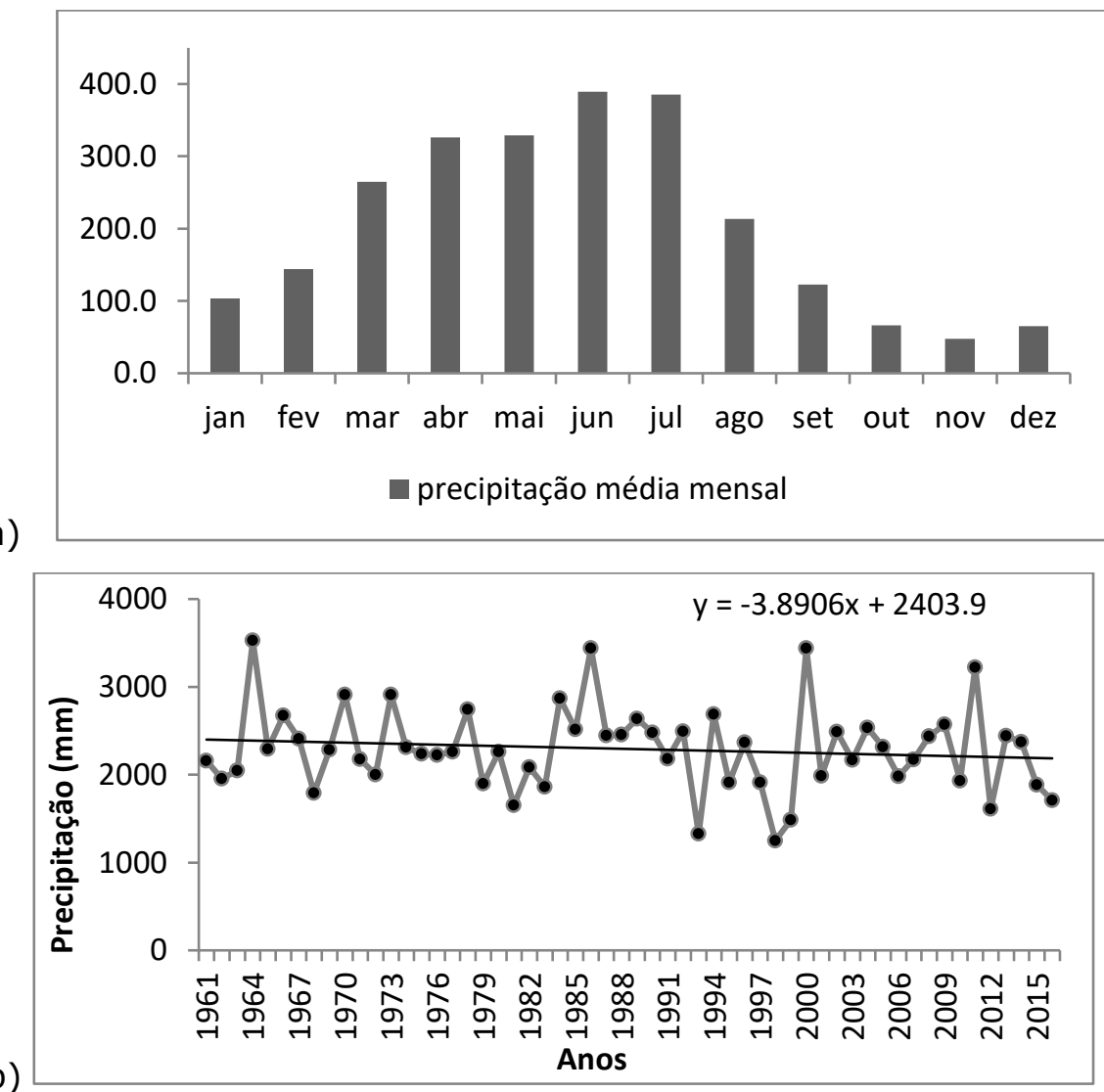

Figura 2 - (a) precipitação média mensal para a cidade de Recife entre 1961-2016. (B) Valores anuais de precipitação para a cidade de Recife entre 1961-2016. Organizado pelos autores. 


\section{CLIMATOLOGIA DOS EVENTOS EXTREMOS DIÁRIOS}

Na figura 03, a qual apresenta os máximos valores diários registrados na série histórica de precipitação (1961-2016), é possível constatar que o quantil diário de chuva extremamente forte $(X>50 \mathrm{~mm})$ é alcançado em todos os anos da série. Chuvas diárias iguais ou superiores a $100 \mathrm{~mm}$ também possuem frequência elevada, sendo observadas em 41 dos 56 anos de registros considerados.

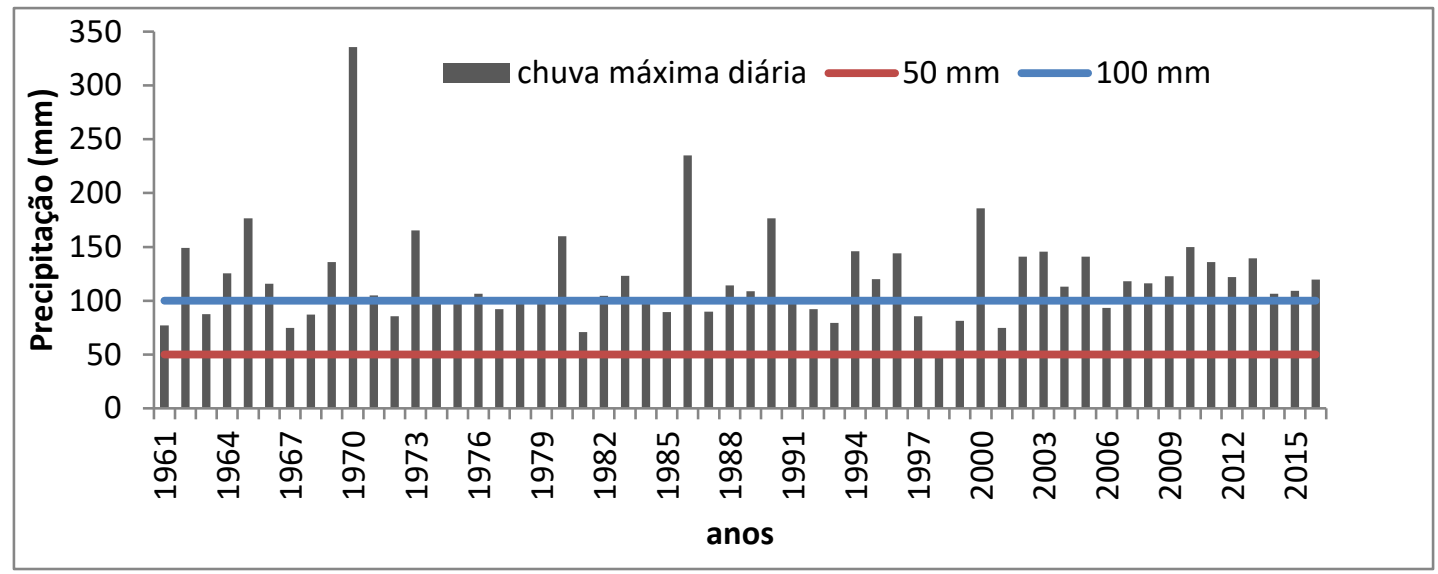

Figura 3 - máximos valores diários registrados por ano entre os anos de 1961 e 2016 na estação do Curado, em Recife. Fonte: INMET. Org. dos autores.

Na tabela 02 podem ser observados os dez maiores volumes diários de precipitação registrados entre 1961 e 2016 na estação do INMET. Destacam-se valores excepcionais como os observados no dia 11 de agosto de 1970 (335,8 $\mathrm{mm}$ ) e no dia 24 de maio de 1986 (235 mm). Tendo conhecimento dos impactos relacionados a esses eventos, considerar a ciclicidade ou tempo de retorno desses fenômenos é uma estratégia fundamental para o planejamento urbano.

Tabela 2 - dez maiores eventos de precipitação diária para a série climatológica de 1961-2016 da cidade do Recife

\begin{tabular}{cc}
\hline Precipitações máximas diárias registradas na cidade \\
do Recife
\end{tabular}

Fonte: organizado pelos autores. Fonte: INMET. Org. dos autores. 


\section{ANÁLISE DE FREQUÊNCIA DOS EVENTOS EXTREMOS}

A climatologia dos eventos extremos de precipitação diária para a cidade do Recife (1961-2016) demonstra que as maiores frequências absolutas dos quantis de chuva forte a extremamente forte estão concentrados entre os meses de março e julho (Figura 04). Os meses de junho e julho são aqueles em que é registrada a maior ocorrência desses eventos, com 269 e 250 eventos iguais ou superiores a $25 \mathrm{~mm}$, respectivamente. Já os meses de outubro a dezembro são aqueles com menor registro de chuvas diárias muito fortes $(25<\mathrm{X}<50 \mathrm{~mm}$ ) ou extremamente fortes $(X>50 \mathrm{~mm})$.

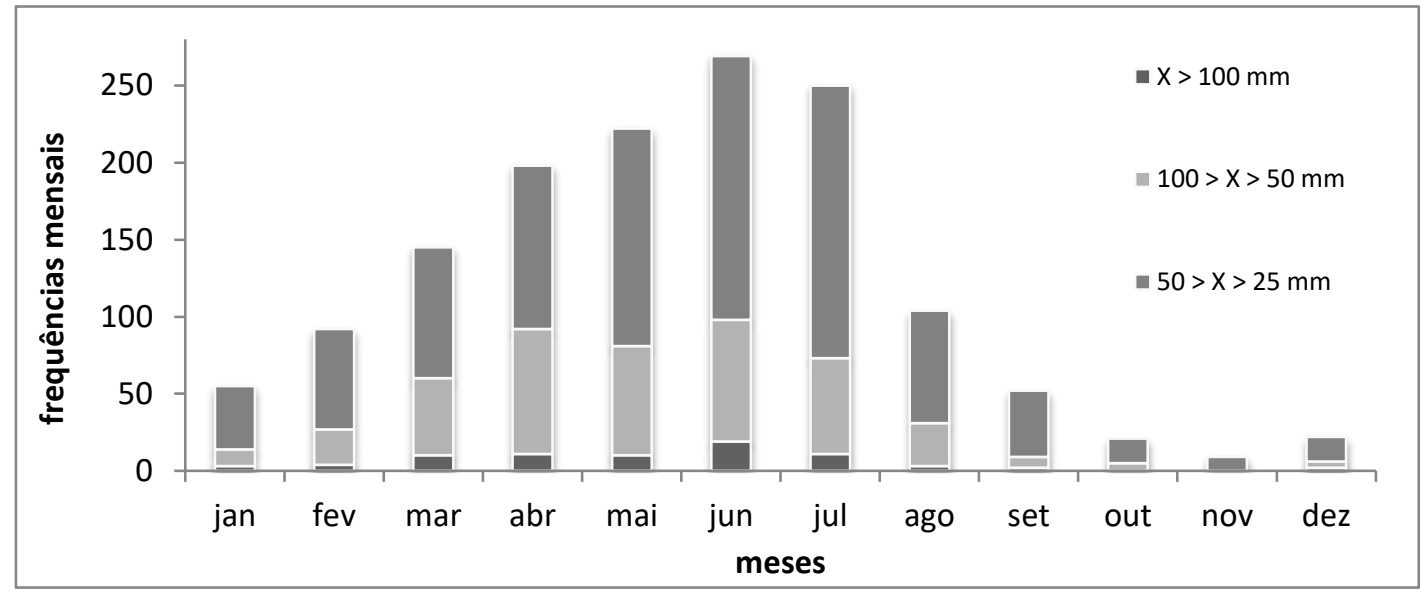

Figura 4 - frequências mensais dos eventos pluviométricos nas faixas dos quantis de chuva diária muito forte $(Q p=0,85)$, extremamente forte $(Q p=0,95)$ e excepcionalmente forte $(2 * Q p=0,95)$ entre os anos de 1961 e 2016 na cidade do Recife/PE. Fonte: INMET. Org.dos autores.

O teste de Mann-Kendall demonstrou que não há tendência significativa em relação à frequência de ocorrência anual dos eventos pluviométricos diários nos intervalos $25<X<50 \mathrm{~mm}$ (Figura 05) e $50<X<100 \mathrm{~mm}$ (Figura 06). Observa-se que há uma variabilidade interanual expressiva dos valores de ocorrência anual para ambos os intervalos de chuvas citados anteriormente. Os volumes diários de chuva iguais ou superiores a $25 \mathrm{~mm}$ e inferiores a $50 \mathrm{~mm}$ possuem uma média de ocorrência de 33 eventos por ano, já os superiores ou iguais a $50 \mathrm{~mm}$ possuem uma média de ocorrência de sete eventos por ano. As máximas frequências anuais de eventos iguais ou superiores a $25 \mathrm{~mm}$ e inferiores a $50 \mathrm{~mm}$ foram registradas nos anos de 1964 (54), 1986 (60), 2009 (56) e 2013 (52). Para os eventos diários iguais ou superiores a $50 \mathrm{~mm}$ as máximas ocorrências foram observadas nos anos de 1964 (15), 1966 (13), 1967 (13), 1970 (12), 1978 (12), 1984 (12), 1985 (12), 2008 (11) e 2011 (16). 


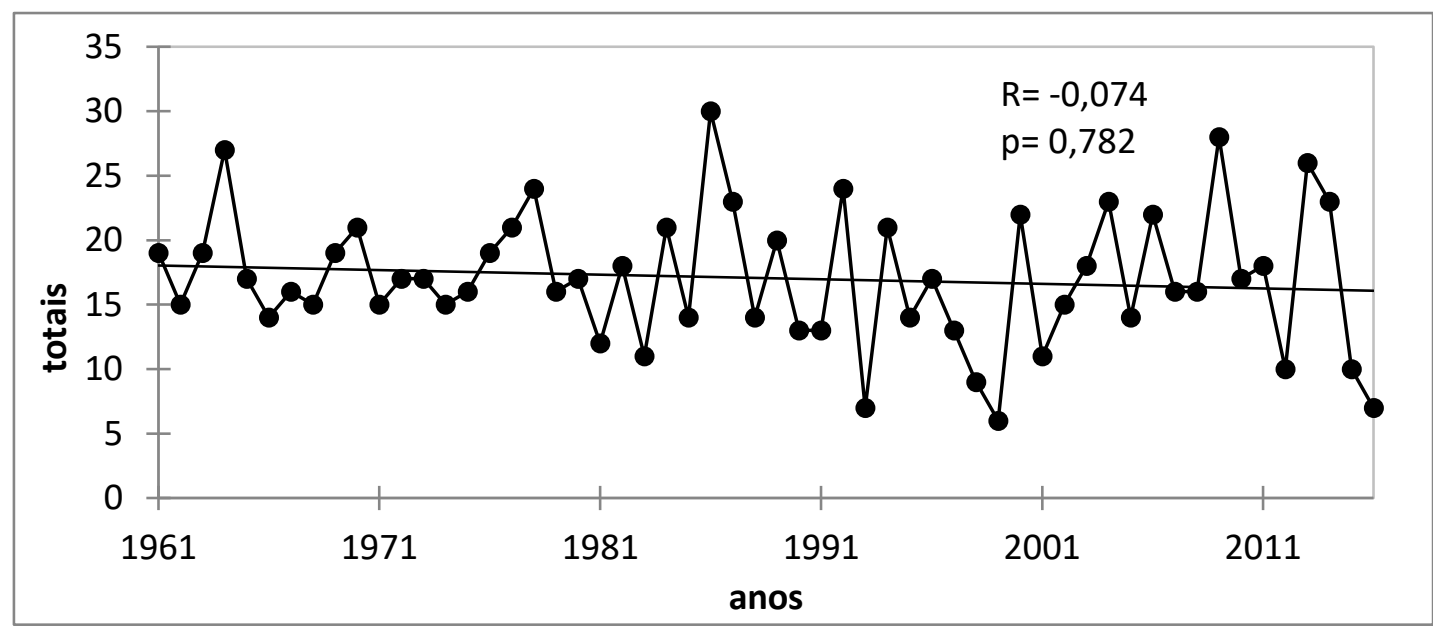

Figura 5 - Frequências anuais de ocorrência de precipitação diária no quantil de chuva muito forte $(Q p=0,85)$ entre 1961 e 2016 para a cidade do Recife. Org. dos autores.

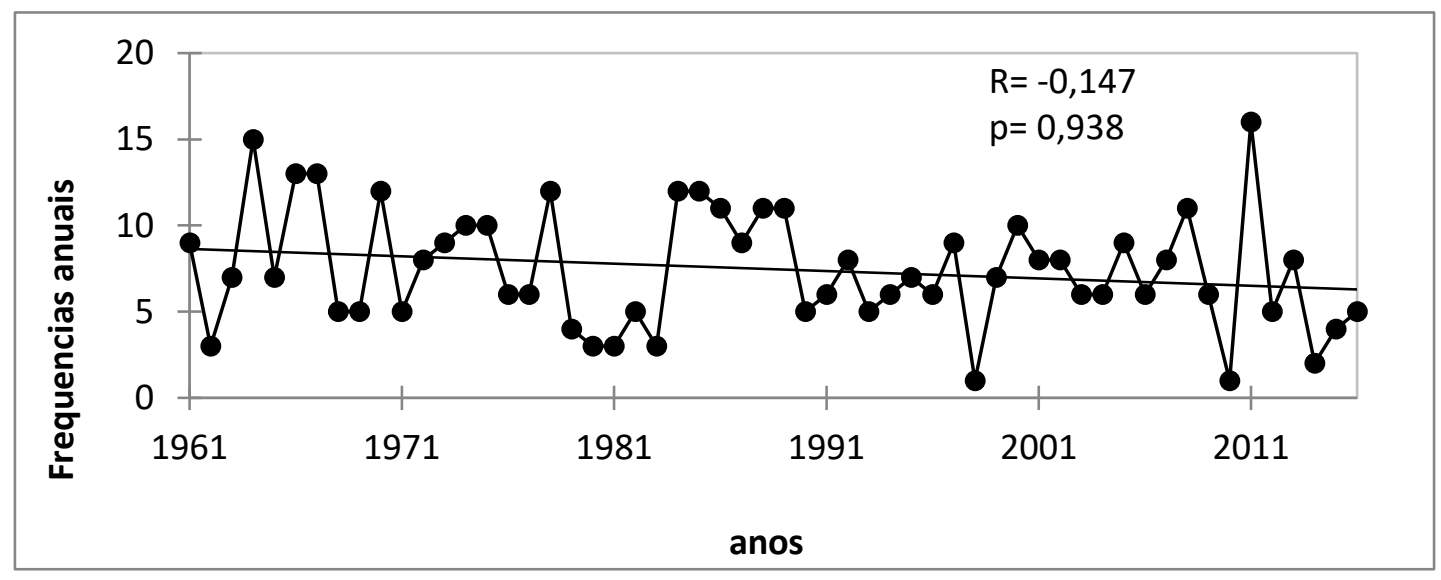

Figura 6 - Frequências anuais de ocorrência de precipitação diária no quantil de chuva extremamente forte $(Q p=0,95)$ entre 1961 e 2016 para a cidade do Recife. Org. dos autores.

Para os eventos diários de precipitação maiores ou iguais a $100 \mathrm{~mm}$ foi constatada, através do teste de Mann Kendall, uma fraca tendência positiva na frequência anual. A média de ocorrência anual dessa classe de precipitação diária é de um evento por ano. Todavia, é possível observar, a partir da Figura 07, a existência de uma alta variabilidade das ocorrências anuais desses eventos extremos diários. Constata-se também uma mudança de padrão de distribuição anual referente a esses eventos de precipitação extrema na série temporal estudada.

Entre 1961 e 2001 pode ser identificado um primeiro ciclo, foi verificada uma recorrência média de 2,3 anos sem registro de eventos iguais ou superiores a $100 \mathrm{~mm}$, associada a uma recorrência média de 8,5 anos com registro de quatro eventos diários de chuvas dessa magnitude. Em um segundo ciclo, delimitado entre 2002 e 2016, foi registrado apenas um ano sem ocorrência de 
eventos diários iguais ou superiores a 100 mm, com uma recorrência de 1,6 anos para registro de pelo menos dois eventos.

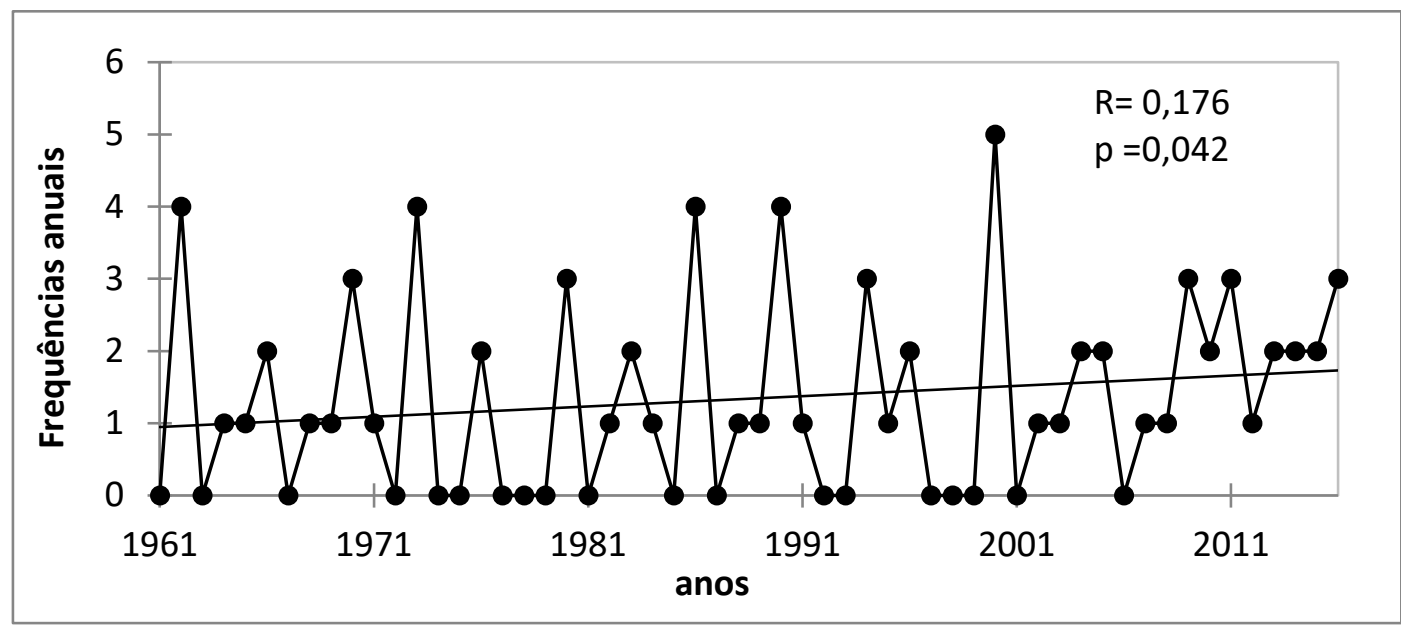

Figura 7 - Frequências anuais de ocorrência de precipitação diária para o dobro do quantil de chuva excepcionalmente forte $(2 * Q p=0,95)$ entre 1961 e 2016 para a cidade do Recife. Org. dos autores.

\section{TEMPO DE RETORNO dOS EVENTOS EXTREMOS DE PRECIPITAÇÃo DIÁRIA}

Os resultados do teste Kolgomorov-Smirnov (Tabela 03) indicam que a amostra é representativa das precipitações extremas do local estudado. A divergência máxima entre as probabilidades de Gumbel e empírica para os máximos pluviométricos anuais atende a todos os intervalos de significância para a estimativa. Nas distribuições mensais, aqueles com divergência máxima menos satisfatória foram os meses de agosto, outubro e dezembro, os quais atenderam aos níveis de significância de $5 \%$ e $1 \%$. Para os demais meses, as probabilidades apresentaram divergência máxima satisfatória a todos os níveis de significância.

A avaliação da qualidade do ajuste pela função $R^{2}$ demonstra que os valores oscilaram entre 0,932 e 0,992 para o método da regressão linear múltipla. Dessa maneira, pode-se considerar que esses resultados indicam boa aderência, pois os valores estão próximos do limite máximo. 
Tabela 3 - Resultado do teste K-S entre as distribuições Gumbel e Empírica.

\begin{tabular}{|c|c|c|c|c|c|c|}
\hline \multirow{2}{*}{$\begin{array}{c}\text { Escala } \\
\text { temporal } \\
\text { da } \\
\text { distribuição } \\
(1961- \\
2016) \\
\end{array}$} & \multirow[t]{2}{*}{$\begin{array}{l}\text { Divergência } \\
\text { máxima } \\
\text { (D) }\end{array}$} & \multicolumn{4}{|c|}{$\begin{array}{l}\text { Valores críticos do teste K-S } \\
\text { e Níveis de significância }\end{array}$} & \multirow[t]{2}{*}{$\begin{array}{l}\text { Qualidade } \\
\text { do ajuste } \\
\left(\mathbf{R}^{2}\right)\end{array}$} \\
\hline & & $20 \%$ & $10 \%$ & $5 \%$ & $1 \%$ & \\
\hline Anual & 0,0798 & 0,1429 & 0,1630 & 0,1817 & 0,2178 & 0,991 \\
\hline Janeiro & 0,0999 & 0,1429 & 0,1630 & 0,1817 & 0,2178 & 0,978 \\
\hline Fevereiro & 0,0946 & 0,1429 & 0,1630 & 0,1817 & 0,2178 & 0,979 \\
\hline Março & 0,1047 & 0,1429 & 0,1630 & 0,1817 & 0,2178 & 0,979 \\
\hline Abril & 0,0945 & 0,1429 & 0,1630 & 0,1817 & 0,2178 & 0,984 \\
\hline Maio & 0,0552 & 0,1429 & 0,1630 & 0,1817 & 0,2178 & 0,990 \\
\hline Junho & 0,0667 & 0,1429 & 0,1630 & 0,1817 & 0,2178 & 0,991 \\
\hline Julho & 0,0667 & 0,1429 & 0,1630 & 0,1817 & 0,2178 & 0,992 \\
\hline Agosto & 0,1786 & 0,1429 & 0,1630 & 0,1817 & 0,2178 & 0,955 \\
\hline Setembro & 0,0967 & 0,1429 & 0,1630 & 0,1817 & 0,2178 & 0,978 \\
\hline Outubro & 0,1679 & 0,1429 & 0,1630 & 0,1817 & 0,2178 & 0,932 \\
\hline Novembro & 0,0918 & 0,1429 & 0,1630 & 0,1817 & 0,2178 & 0,982 \\
\hline Dezembro & 0,1708 & 0,1429 & 0,1630 & 0,1817 & 0,2178 & 0,945 \\
\hline
\end{tabular}

Org. dos autores.

Na Figura 08 estão representados os diagramas de dispersão, os quais relacionam as probabilidades do modelo de Gumbel e as probabilidades empíricas mensais para os máximos eventos diários de precipitação. É possível reforçar a existência de aderência razoável entre as probabilidades comparadas. 


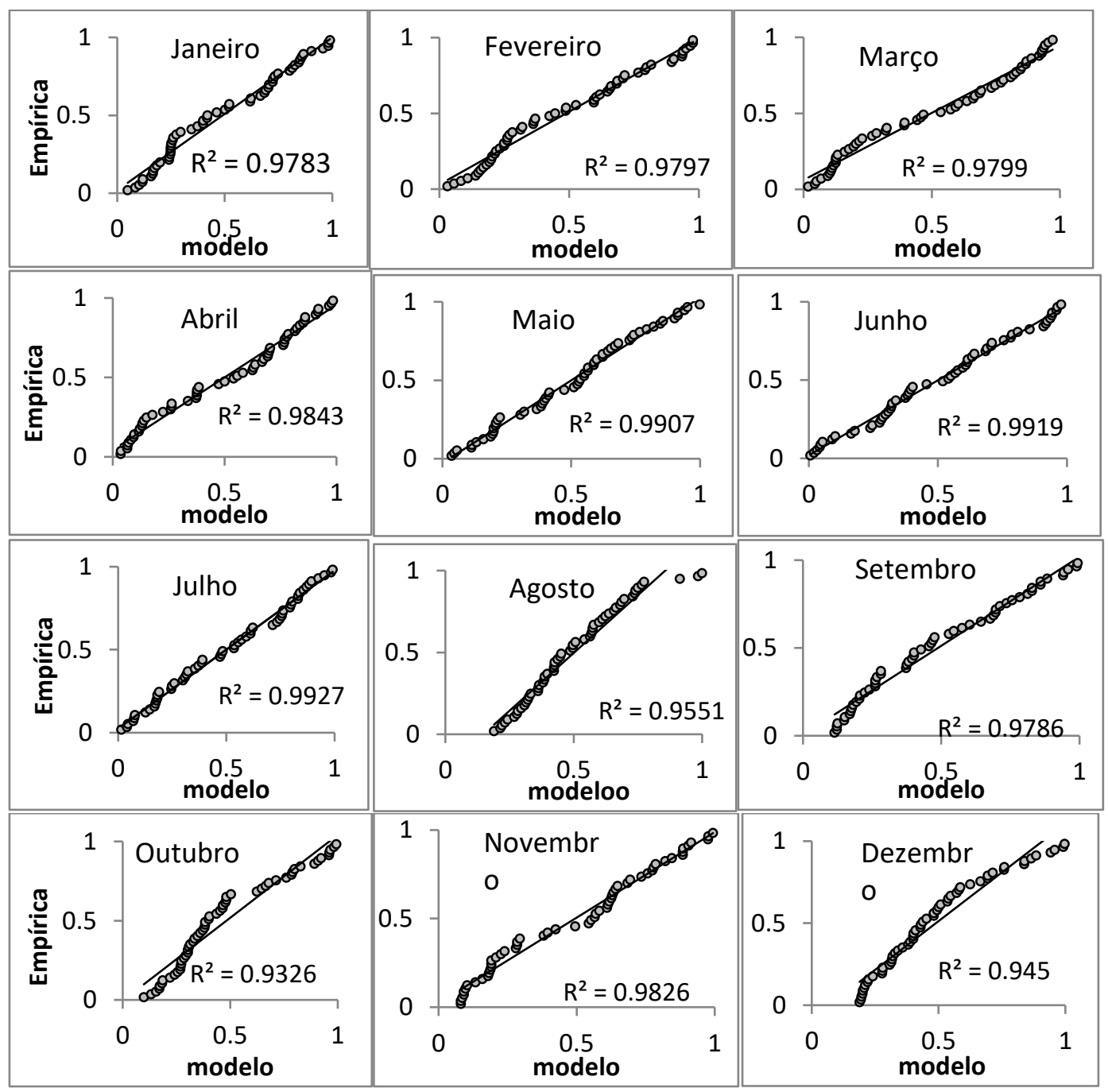

Figura 8 - Gráficos de dispersão das probabilidades de Gumbel e empírica para ocorrência de eventos máximos de precipitação diária por mês (1961-2016). Org. dos autores.

A distribuição de Gumbel para os máximos valores diários de precipitação, registrados por ano, (Figura 09(a)) revela um tempo de retorno de dois anos para eventos de $112,7 \mathrm{~mm}$, de cinco anos para eventos de $151,9 \mathrm{~mm}$, de dez anos para eventos $177,9 \mathrm{~mm}$ e vinte anos para eventos de $202,8 \mathrm{~mm}$. De modo complementar, o gráfico de probabilidade de ocorrência de eventos diários de precipitação (figura 09 (b)) revela que um evento com volume de $71 \mathrm{~mm}$ possui $90 \%$ de chance de ocorrer a cada ano, um com volume de $102,9 \mathrm{~mm}$ possui chance de $60 \% /$ ano, um com volume de $135,6 \mathrm{~mm}$ possui chance de $30 \%$ /ano e um com volume de $177,8 \mathrm{~mm}$ possui chance de $10 \% /$ ano. Esses dados corroboram a importância dos valores extremos de chuva na climatologia da cidade do Recife. Esses resultados demonstram que o quantil de precipitação diária extremamente forte $(100>X>50 \mathrm{~mm})$ possui uma recorrência anual.

Em estudo realizado por Silva et al.(2013) foram encontrados tempo de retorno de eventos máximos pluviais diários por ano que corroboram os resultados do presente estudo. Na escala temporal diária esses autores 
estimaram um tempo de retorno de cinco anos para precipitação de 150 $\mathrm{mm} /$ dia, de 10 anos para evento pluviométrico de $170 \mathrm{~mm} / \mathrm{dia}$, de cinquenta anos para volume de $235 \mathrm{~mm} /$ dia e de cem anos para evento de $234 \mathrm{~mm} / \mathrm{dia}$.

No município de Cruz das Almas, localizado no estado da Bahia (Brasil) foram estimados tempos de retorno de eventos de precipitação de 112,5 $\mathrm{mm} /$ dia em doze anos, enquanto que para a estação do INMET, no Recife, esse mesmo valor possui recorrência de dois anos. Para o município de Piracicaba, no estado de São Paulo, Sansigolo (2008) encontrou valor de precipitação de 95 $\mathrm{mm} /$ dia com 10 anos de tempo de retorno. Na cidade de Lavras-MG, Beijo et al. (2005) calcularam tempo de retorno de vinte anos para evento pluvial de 87 $\mathrm{mm} /$ dia. Na cidade de Blumenau-SC, Cordero et al.,(2008) verificaram consonância entre volume de chuva de $131,8 \mathrm{~mm} /$ dia e um tempo de retorno de dez anos. Esses dados permitem averiguar a alta recorrência de máximos volumes diários de precipitação, registrados por ano, no sítio urbano do Recife.

(a)
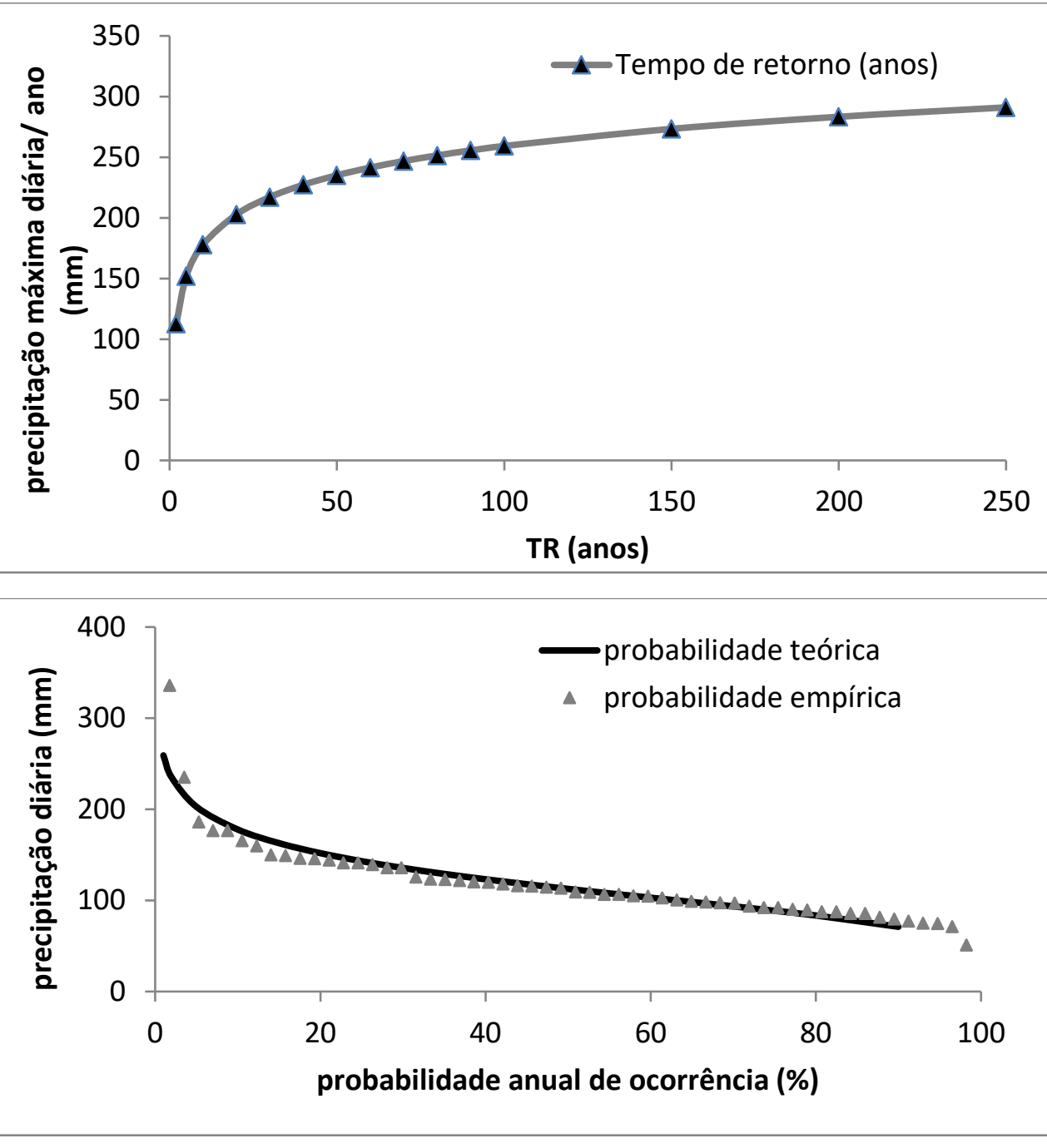

(b)

Figura 9 - (a) gráfico do tempo de retorno dos eventos de precipitação máxima diária/ano para a cidade do Recife, (b) gráfico de probabilidade de ocorrência anual de eventos extremos diários de precipitação na cidade do Recife.Org. dos autores. 
O tempo de retorno dos eventos de máxima precipitação diária por mês, apresentados na Tabela 04, revelam que todos os meses do ano estão sujeitos à ocorrência de chuvas muito fortes $(50>x>25 \mathrm{~mm})$ e extremamente fortes $(100>x>50 \mathrm{~mm})$. Durante os meses de março a agosto volumes diários de alta magnitude possuem maior frequência, quando comparados aos demais meses do ano. O mês de junho, climatologicamente mais chuvoso, se destaca por apresentar mais alta recorrência de máximos pluviométricos diários. Para o mês de junho é estimado um tempo de retorno de cinco anos para eventos de $111,7 \mathrm{~mm}$, de dez anos para eventos de 133,1 $\mathrm{mm}$ e de vinte anos para eventos de 153,7 mm. Já o mês de novembro, menos chuvoso do ano, apresenta os maiores períodos de retorno para eventos extremos de precipitação diária, para esse mês o tempo de retorno de um volume de 32,2 $\mathrm{mm}$ é estimado em vinte anos.

A maior recorrência de eventos extremos entre os meses de março a agosto pode ser explicada pela atuação frequente de sistemas sinóticos convectivos, entre os quais se destacam os Distúrbios Ondulatórios de Leste e a Zona de Convergência Intertropical (MACHADO et al., 2012; MOLION e BERNARDO, 2002).

Tabela 4 - tempo de recorrência dos máximos eventos diários de precipitação por mês na cidade do Recife.

\begin{tabular}{cccccccccccccc}
\hline \multicolumn{10}{c}{ Precipitação máxima diária mensal (mm) } \\
\hline $\begin{array}{c}\text { TR* } \\
\mathbf{2}\end{array}$ & jan & $\mathbf{f e v}$ & $\mathbf{m a r}$ & $\mathbf{\text { abr }}$ & $\mathbf{m a i}$ & jun & jul & ago & set & out & nov & dez \\
$\mathbf{5}$ & 53,8 & 66,6 & 88,7 & 91 & 94,7 & 111,7 & 94,1 & 86,3 & 47,8 & 34,4 & 21 & 44,3 \\
$\mathbf{1 0}$ & 69,3 & 84,9 & 109,5 & 111,9 & 115,5 & 133,1 & 113,6 & 114,5 & 61 & 45,5 & 26,7 & 59,4 \\
$\mathbf{2 0}$ & 84,2 & 102,4 & 129,5 & 131,9 & 135,3 & 153,7 & 132,2 & 141,5 & 73,7 & 56,1 & 32,2 & 73,9 \\
$\mathbf{5 0}$ & 103,5 & 125,1 & 155,3 & 157,8 & 161,1 & 180,3 & 156,4 & 176,5 & 90,2 & 69,8 & 39,3 & 92,7 \\
$\mathbf{1 0 0}$ & 177,9 & 142,1 & 174,7 & 177,3 & 180,4 & 200,2 & 174,5 & 202,7 & 102,5 & 80,1 & 44,6 & 106,7 \\
$\mathbf{1 5 0}$ & 126,3 & 152 & 185,9 & 188,6 & 191,6 & 211,8 & 185,1 & 218 & 109,7 & 86,1 & 47,7 & 114,9 \\
$\mathbf{2 0 0}$ & 132,3 & 159 & 193,9 & 196,6 & 199,6 & 220,1 & 192,6 & 228,9 & 114,8 & 90,3 & 49,9 & 120,7 \\
\hline
\end{tabular}

Fonte: INMET. Org. dos autores. * TR= tempo de retorno em anos.

A comparação entre estimativas do tempo de retorno de máximas precipitações diárias em outros locais de clima tropical e subtropical permite afirmar que a cidade do Recife apresenta máximos pluviométricos diários elevados, com tempos de retorno relativamente curtos. Nesse sentido, as precipitações diárias intensas correspondem a importantes fluxos de entrada de energia na paisagem urbana da cidade, correspondendo a um aspecto chave no planejamento do referido espaço urbano. 


\section{CONCLUSÕES}

A investigação das frequências anuais dos eventos de precipitações extremas diárias, pelo método de Mann-Kendall, não demonstrou tendências significativas. A distribuição de Gumbel demonstrou estimativas satisfatórias de probabilidade e tempo de retorno de eventos pluviais extremos. A partir do teste de Kolgomorov-Smirnov pode-se verificar a existência de aderência satisfatória entre as probabilidades obtidas pelo modelo de Gumbel e aquelas calculadas empiricamente.

A distribuição demonstrou que para os eventos pluviométricos diários máximos anuais, foram estimados tempos de retorno de dois anos para eventos diários de 112,7 mm, cinco anos para eventos de 151,9 mm, dez anos para eventos de $177,9 \mathrm{~mm}$ e vinte anos para eventos de $202,8 \mathrm{~mm}$. Para o tempo de recorrência mensal, a distribuição demonstrou que todos os meses do ano são propícios a ocorrência de valores diários extremos, sendo que o período entre março e agosto apresenta maior probabilidade.

Portanto, os resultados apresentados reforçam a suscetibilidade da área de estudo aos eventos extremos de chuvas diárias, devendo esse aspecto ser considerado um fator importante no planejamento territorial da cidade do Recife.

\section{REFERÊNCIAS}

ARNBJERG-NIELSEN,K., WILLEMS, P. OLSSON, J. BEECHAM, S., PATHIRANA, A., BULOW GREGERSEN, I., MADSEN, H., NGUYEN, V. Impacts of climate change on rainfall extremes and urban drainage systems: a review. Water Science \& Technology.v.68, p.16-28, 2013.

BARROS, H.R., LOMBARDO, M. A. Zoneamento climático urbano da cidade do Recife: uma contribuição ao planejamento urbano. GEOUSP-espaço e tempo. v.1, n33, 2013.

BEIJO, A.L.; MUNIZ, J.A.; NETO, P.C. Tempo de retorno das precipitações máximas em Lavras (MG) pela distribuição de valores extremos tipo I. Revista de Ciências Agrotécnicas, v.29, n.3, p.657-667, 2005.

CORDERO, A.; SEVERO, D.L.; MEDEIROS, H.S.S.; TACHINI, M.;MEDEIROS, P.A. Estudo da precipitação máxima diária para Blumenau-SC e o evento de novembro de 2008. Anais do XVIII Simpósio de Recursos Hídricos, 2008.

CORREA, A.C.B. Contribuição à análise do Recife como um geossistema urbano. Revista de Geografia (UFPE). v.23, n.3, 2006.

DUARTE, C.C.; NÓBREGA, R.S.; COUTINHO, R.Q. Análise climatológica e dos eventos extremos de chuva no município de Ipojuca, Pernambuco. Revista de Geografia (UFPE). v.32, n. 2, p.158-176, 2015.

FARIAS, R. F. L; ALVES, K. M. A. S.; NÓBREGA, R. S. Climatologia da ocorrência de eventos extremos de precipitação na mesorregião do Sertão Pernambucano. Revista Geonordeste, Edição Especial 2, v. 1, n. 5, p. 930-941, 2012.

GOCIC, M., TRAJKOVIC, S. Analysis of precipitation and drought data in Serbia over the period 1980-2010. Journal of Hydrology. V.494, p.32- 42, 2013. 
MACHADO, C.C.C.; NÓBREGA, R.S.; OLIVEIRA, T.H.; ALVES, K.M.A. Distúrbio Ondulatório de Leste como condicionante a eventos extremos de precipitação em Pernambuco. Revista Brasileira de Climatologia. V.11, n.8, p.146-188, 2012.

MENDONÇA, F.; DANNI-OLIVEIRA, I. M. Climatologia: noções básicas e climas do Brasil. São Paulo: Oficina de Textos, 2007. 206p.

MOLION, L.C.B.; BERNARDO, S.O. Uma revisão da dinâmica das chuvas no Nordeste Brasileiro. Revista Brasileira de Meteorologia, v.17, n.1, p. 1-10, 2002.

MOTA, G.V. Estudo observacional de distúrbios ondulatórios de leste no nordeste brasileiro. 1997. 93p. Dissertação (Mestrado em Meteorologia)-Instituto Astronômico e Geofísico, Universidade São de Paulo, São Paulo, 1997.

SANSIGOLO, C.A. Distribuição de extremos de precipitação diária, temperatura máxima e mínima e velocidade do vento em Piracicaba, SP (1917-2006). Revista Brasileira de Meteorologia. v.23, n.3, p.341-346, 2008.

SILVA, S.R.; ARAÚJO, G.R.S. Algoritmo para a determinação de equação de chuvas intensas. Revista Brasileira de Geografia Física.V.6, n.5, p.1371-1383, 2013.

SOUZA, W. M.; AZEVEDO, P.V.; ARAÚJO, L.E. Classificação diária e impactos decorrentes dos desastres associados às chuvas na cidade do Recife-PE. Revista Brasileira de Geografia Física. v.2,p.250-268, 2012.

XAVIER, T. M. B. S. A técnica dos quantis e suas aplicações em meteorologia, climatologia e hidrologia, com ênfase para as regiões brasileiras. Brasília: Thesaurus, 2002. $140 \mathrm{p}$.

ZHANG, Q.; CHEN, X.; STEFAN, B. Spatio-temporal variations of precipitation extremes in Yangtze River Basin (1960-2012), China. Atmospheric and climate Sciences. v.1, p.1-18, 2011.

ZAHIRI, E.P; BAMBA, I.;FAMIEN, A.M.; KOFFI, A.K.; OCHOU, A.D. Mesoescale extreme rainfall events in West Africa: the case of Niamey (Niger) and Upper Ouémé Valley (Benin). Weather and climate extremes. v.13, p.15-34, 2016. 2. 